EESTI NSV TEADUSTE AKADEEMIA TOIMETISED. 25. KOIDE FUUSIKA * MATEMAATIKA. 1976, NR. 3

ИЗВЕСТИЯ АКАДЕМИИ НАУК ЭСТОНСКОН ССР. ТОМ 25 ФНЗНКА * МАТЕМАТИКА. 1976, № 3

\title{
ОБОСНОВАНИЕ СПЕЦИАЛЬНОЙ ТЕОРИИ ОТНОСИТЕЛЬНОСТИ БЕЗ ВТОРОГО ПОСТУЛАТА ЭИНШТЕЙНА
}

Развит в двух вариантах метод построения специальной теории относительности, использующий, наряду с отказом от классической теории, только принцип относительности и, в одном из вариантов, законы сохранения массы и импульса. Принцип постоянства скорости света получается как одно из первых следствий этих предпосылок, но не берется в качестве второго исходного постулата. Новый метод не нуждается и в требовании групповости инерциальных преобразований, отличаясь в этом отношении от известных до сих пор других методов, тоже не использующих второго постулата Эйнштейна.

\section{Введение}

Впервые в $\left[^{1-4}\right]$ было показано, что при выводе преобразований Лоренца второй постулат Эйнштейна может быть заменен требованием групповости преобразований из одной инерциальной системы в другую. Это требование заключается в том, что если имеем три инерциальные системы, то преобразование координат и времени из первой системы во вторую, а из второй в третью должно дать результат, тождественный с результатом преобразования тех же величин прямо из первой системы в третью. В $\left[^{5}\right]$ был развит аналогичный метод, не использующий преобразований Лоренца. Вместо этого требовалось, чтобы последовательное преобразование скорости тела из первой инерциальной системы во вторую, а из второй в третью давало результат, одинаковый с получаемым при непосредственном переходе из первой системы в третью.

Легко видеть, что требование групповости инерциальных преобразований является - безразлично, в какой форме - слишком сильным. Групповые свойства преобразований - будь то координат и времени, будь то скорости - являются фактически чем-то само собою разумеющимся. В самом деле, отказ от этого требования означал бы явную нелепость - одно и то же тело имело бы, например, в одной и той же инерциальной системе две или даже много различных скоростей. Отсюда возникает мысль, что требование групповости излишне, что все те же результаты должно быть возможно получить и помимо этого требования. В таком случае в роли единственного основного постулата специальной теории относительности выступил бы только принцип относительности. Второй постулат Эйнштейна получался бы отсюда как прямое следствие, без привлечения групповых соображений. В настоя- 
щей статье покажем, что такое построение теории относительности действительно возможно, и притом по меньшей мере двумя различными, хотя во многом аналогичными способами. Изложим их по порядку.

\section{Метод, основанный на законах сохранения массы и импульса}

Кроме принципа относительности, в развиваемом здесь методе используются законы сохранения массы и импульса с учетом возможной зависимости массы от скорости.

Рассмотрим полностью неупругое соударение двух тел, имеющих массы покоя $m_{1}$ и $m_{2}$. В результате соударения образуется вторичное тело с массой покоя $m_{3}$. Каждое из этих трех тел можно рассматривать как покоящееся, измеряя относительно него скорости двух других тел. Обозначим через $v_{i k}$ скорость $k$-го тела относительно $i$-го. В силу симметрии и равноправия всех инерциальных систем, очевидно, $v_{k i}=-v_{i k}$, а в силу сохранения импульса все скорости коллинеарны. Множитель, определяющий возможную зависимость массы от скорости, обозначим через $\gamma(v)$, причем из соображений симметрии $\gamma(-v)=\gamma(v) ; \gamma\left(v_{i k}\right)$ будем кратко писать как $\gamma_{i k}$.

Теперь запишем равенства, выражающие сохранение массы и импульса во всех трех инерциальных системах - в системах покоя каждого из трех тел. В системе покоя 1-го тела

$$
\begin{aligned}
& m_{1}+m_{2} \gamma_{12}=m_{3} \gamma_{13}, \\
& m_{2} v_{12} \gamma_{12}=m_{3} v_{13} \gamma_{13} .
\end{aligned}
$$

В системе покоя 2-го тела

$$
\begin{aligned}
& m_{1} \gamma_{12}+m_{2}=m_{3} \gamma_{32}, \\
& m_{1} v_{12} \gamma_{12}=m_{3} v_{32} \gamma_{32} .
\end{aligned}
$$

В системе покоя 3-го тела

$$
\begin{aligned}
& m_{1} \gamma_{13}+m_{2} \gamma_{32}=m_{3}, \\
& m_{1} v_{13} \gamma_{13}=m_{2} v_{32} \gamma_{32} .
\end{aligned}
$$

Отметим, что уравнения (2), (4) и (6), выражающие сохранение импульса, не независимы - каждое из них, независимо от вида функции $\gamma(v)$, является следствием двух других. Из тех же уравнений видно, что скорости $v_{12}, v_{13}$ и $v_{32}$ имеют одинаковый знак, т. е. направлены в одну и ту же сторону. 'Будем для определенности считать все их положительными, хотя для дальнейших рассуждений это несущественно. Из уравнений (1) и (2) следует также, что $v_{13}<v_{12}$, а из уравнений (3) и (4) - что $v_{32}<v_{12}$. Наоборот, скорости $v_{13}$ и $v_{32}$ могут быть, в зависимости от масс, в любом соотношении друг с другом, т. е. $v_{13} \lessgtr v_{32}$.

Чтобы вывести из уравнений $(1)-(6)$ вид функции $\gamma(v)$ и формулу сложения скоростей, исключим прежде всего массы. Это удобнее всего сделать так. Исключая из уравнений (1) и (2) $m_{2}$ или $m_{3}$, получаем:

$$
\begin{aligned}
& m_{1}=m_{3} \gamma_{13}\left(1-v_{13} / v_{12}\right), \\
& m_{1}=m_{2} \gamma_{12}\left(-1+v_{12} / v_{13}\right) .
\end{aligned}
$$

Точно так же, исключая из уравнений (3) и (4) $m_{1}$ или $m_{3}$, имеем:

$$
m_{2}=m_{3} \gamma_{32}\left(1-v_{32} / v_{12}\right),
$$




$$
m_{2}=m_{1} \gamma_{12}\left(-1+v_{12} / v_{32}\right) .
$$

Наконец, из уравнений. (5) и (6), исключая $m_{1}$ или $m_{2}$, находим:

$$
\begin{aligned}
& m_{3}=m_{2} \gamma_{32}\left(1+v_{32} / v_{13}\right), \\
& m_{3}=m_{1} \gamma_{13}\left(1+v_{13} / v_{32}\right) .
\end{aligned}
$$

Далее перемножим полученные шесть уравнений попарно: (7) и (12), (8) и $(10),(9)$ и (11). Тогда все массы сократятся и мы получим:

$$
\begin{aligned}
& \left(1-v_{13} / v_{12}\right)\left(1+v_{13} / v_{32}\right) \gamma_{13}^{2}=1, \\
& \left(1-v_{12} / v_{13}\right)\left(1-v_{12} / v_{32}\right) \gamma_{12}^{2}=1, \\
& \left(1-v_{32} / v_{12}\right)\left(1+v_{32} / v_{13}\right) \gamma_{32}^{2}=1 .
\end{aligned}
$$

Если теперь исключим из уравнений (13) и (14) $v_{32}$, то найдем

$$
\left(1-\gamma_{12}^{-2}\right) v_{12}^{-2}=\left(1-\gamma_{13}^{-2}\right) v_{13}^{-2} \text {. }
$$

Аналогичные равенства получим еще, исключая $v_{13}$ из уравнений (14) и (15) или $v_{12}$ из уравнений (13) и (15). Окончательный результат таков:

$$
\left(1-\gamma_{12}^{-2}\right) v_{12}^{-2}=\left(1-\gamma_{13}^{-2}\right) v_{13}^{-2}=\left(1-\gamma_{32}^{-2}\right) v_{32}^{-2} .
$$

Каждое из трех выражений зависит здесь одинаковым образом только от одной из трех скоростей. Но две из скоростей независимы друг от друга, что видно, например, из уравнения (6): задав $v_{13}$, мы можем, подбирая произвольно отношение масс $m_{1} / m_{2}$, задать независимо и $v_{32}$. Следовательно, равенство (17) выполнимо только тогда, если все три выражения равны одной и той же постоянной. Если эту постоянную примем равной нулю, то

$$
\gamma(v)=1,
$$

а любое из уравнений $(13)-(15)$ даст

$$
v_{12}=v_{13}+v_{32} \text {. }
$$

Это - нерелятивистские (классические) формулы. Отказ от классической теории требует, чтобы постоянная была отлична от нуля. Очевидно, она должна иметь размерность обратного квадрата скорости. Отсюда вытекает существование универсальной скорости. Здесь возникает еще вопрос о знаке. К теории относительности мы придем, приняв постоянную положительной и отождествив универсальную скорость со скоростью света $c$. Таким образом,

откуда

$$
\left(1-\gamma^{-2}(v)\right) v^{-2}=c^{-2},
$$

$$
\gamma(v)=\left(1-v^{2} / c^{2}\right)^{-1 / 2} \text {. }
$$

Формулу сложения скоростей даст опять любое из уравнений (13)(15), но вместо (19) она получится в известном релятивистском виде:

$$
v_{12}=\left(v_{13}+v_{32}\right)\left(1+v_{13} v_{32} / c^{2}\right)^{-1} .
$$

Итак, мы получили основные формулы теории относительности. Дальнейшее построение теории, как показано в $\left[{ }^{6}\right]$ и $\left[{ }^{7}\right]$, труда не составляет. В частности, инвариантность скорости света можно заключить из преобразований Лоренца, которые вытекают в свою очередь из формул преобразования компонентов скорости. 
Открытой остается еще возможность отрицательной постоянной в формуле (17). Обсуждение ее отложим.

\section{Метод, основанный на сравнении длин движущихся стержней}

Другой способ, приводящий к формуле (17) со всеми вытекающими из нее следствиями, основан на сравнении длин трех параллельных стержней, движущихся друг относительно друга, с учетом возможной зависимости длины от скорости.

Обозначим длины покоя стержней через $l_{1}, l_{2}, l_{3}$, а их относительные скорости через $v_{12}, v_{13}, v_{32}$ (индексы пишем в том же смысле, как и в предыдущем разделе). Длины и скорости выберем так, чтобы последовательно во времени имели место следующие три события: 1) совпадение левого конца 1-го и правых концов 2-го и 3-го стержней; 2) совпадение левых концов всех трех стержней; 3) совпадение левого конца 2-го и правых концов 1-го и 3-го стержней. На рис. 1-3 показано в системах покоя всех трех стержней их взаимное расположение в указанные три момента.

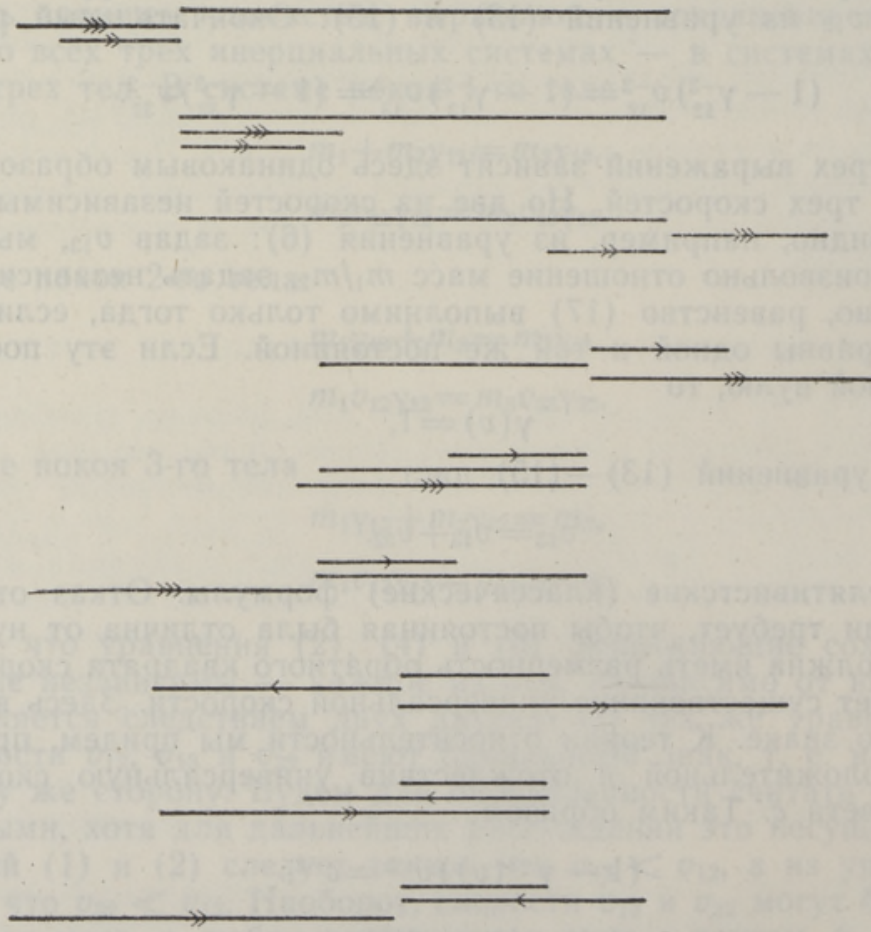

Рис. 1.

На всех рисунках последовательные расположения стержней идут сверху вниз и нумерация стержней идет тоже сверху вниз. Скорости обозначены стрелками: одинарной $v_{32}$, двойной $v_{13}$, тройной $v_{12}$.

Рис. 2.

Рис. 3.

Обозначая множитель, на который нужно умножить длину покоя, чтобы получить длину при движении со скоростью $v$, через $g(v)$ и записывая $g\left(v_{i k}\right)$ как $g_{i k}$, выразим промежутки времени, протекающие между первым и вторым и между вторым и третьим событиями. В системе покоя 1-го стержня (см. рис. 1) промежуток времени между пер- 
вым и вторым событием выражается или как $l_{2} g_{12} v_{12}^{-1}$, или как $l_{3} g_{13} v_{13}^{-1}$, а промежуток времени между вторым и третьим событием как $l_{1} v_{12}^{-1}$ или как $\left(l_{1}-l_{3} g_{13}\right) v_{13}^{-1}$. Итак, имеем два равенства

$$
\begin{gathered}
l_{2} g_{12} v_{12}^{-1}=l_{3} g_{13} v_{13}^{-1}, \\
l_{1} v_{12}^{-1}=\left(l_{1}-l_{3} g_{13}\right) v_{13}^{-1} .
\end{gathered}
$$

Аналогично поступаем в системах покоя 2-го и 3-го стержней (см. рис. 2 и 3) и получаем еще четыре равенства:

$$
\begin{gathered}
l_{2} v_{12}^{-1}=\left(l_{2}-l_{3} g_{32}\right) v_{32}^{-1}, \\
l_{1} g_{12} v_{12}^{-1}=l_{3} g_{32} v_{32}^{-1}, \\
l_{3} v_{13}^{-1}=\left(l_{2} g_{32}-l_{3}\right) v_{32}^{-1}, \\
\left(l_{1} g_{13}-l_{3}\right) v_{13}^{-1}=l_{3} v_{32}^{-1} .
\end{gathered}
$$

Выражая далее из уравнений $(23),(25)$ и (27) отношение длин $l_{2} / l_{3}$, а из уравнений $(24),(26)$ и $(28)$ отношение $l_{3} / l_{1}$, находим:

$$
l_{2} / l_{3}=g_{13} v_{12} / g_{12} v_{13}=g_{32}\left(1-v_{32} / v_{12}\right)^{-1}=g_{32}^{-1}\left(1+v_{32} / v_{13}\right)
$$

и

$$
l_{3} / l_{1}=g_{13}^{-1}\left(1-v_{13} / v_{12}\right)=g_{12} v_{32} / g_{32} v_{12}=g_{13}\left(1+v_{13} / v_{32}\right)^{-1} .
$$

Перемножая эти равенства, находим еще одно отношение длин:

$$
l_{2} / l_{1}=g_{12}^{-1}\left(-1+v_{12} / v_{13}\right)=g_{12}\left(-1+v_{12} / v_{32}\right)^{-1}=g_{13} v_{32} / g_{32} v_{13} .
$$

Из равенства двух последних выражений в (29), двух крайних в (30) и двух первых в (31) следует:

$$
\begin{gathered}
g_{32}^{2}=\left(1+v_{32} / v_{13}\right)\left(1-v_{32} / v_{12}\right), \\
g_{13}^{2}=\left(1-v_{13} / v_{12}\right)\left(1+v_{13} / v_{32}\right) \\
g_{12}^{2}=\left(1-v_{12} / v_{13}\right)\left(1-v_{12} / v_{32}\right)
\end{gathered}
$$

Эти формулы аналогичны формулам (13)-(15). Более того, они тождественны последним по форме и получаются из них заменой $\gamma_{i k} \rightarrow g_{i k}^{-1}$. Поэтому дальнейшие выкладки излишни; результаты получим, сделав ту же замену в результатах предыдущего раздела. Итак, мы приходим или к классической теории с $g(v)=1$ и формулой (19) сложения скоростей, или к релятивистской формуле сокращения длин

$$
l(v)=l\left(1-v^{2} / c^{2}\right)^{1 / 2}
$$

и к формуле (22) сложения скоростей.

\section{Невозможность обратного знака постоянной}

В [ $\left.{ }^{5}\right]$ случай равенства выражений (17) отрицательной постоянной обходится молчанием. Хотя невозможность отрицательного значения довольно очевидна, представляет интерес проследить несколько подроб- 
нее, к каким следствиям ведет подобный выбор. Покажем, что он не может привести к разумной физической теории. Вместо формул (21) и (22) мы получили бы в этом случае формулы

$$
\gamma(v)=\left(1+v^{2} / C^{2}\right)^{-1 / 2}
$$

и

$$
v_{12}=\left(v_{13}+v_{32}\right)\left(1-v_{13} v_{32} / C^{2}\right)^{-1},
$$

где $-C^{2}-$ упомянутая отрицательная постоянная, причем $C$ есть некоторая универсальная скорость, которую, однако, нет оснований отождествлять со скоростью света. Далее методом статьи $\left[{ }^{6}\right]$ мы получили бы вместо преобразований Лоренца формулы преобразования $x$-координаты и времени в виде

$$
\begin{aligned}
& x^{\prime}=(x-v t)\left(1+v^{2} / C^{2}\right)^{-1 / 2}, \\
& t^{\prime}=\left(t+v x / C^{2}\right)\left(1+v^{2} / C^{2}\right)^{-1 / 2} .
\end{aligned}
$$

Иначе, положив

мы имели бы

$$
v / C=\tan \alpha,
$$

$$
\begin{aligned}
x^{\prime} & =x \cos \alpha-C t \sin \alpha, \\
C t^{\prime} & =x \sin \alpha+C t \cos \alpha .
\end{aligned}
$$

Но эти формулы являются формулами преобразования декартовых координат на эвклидовой плоскости. Следовательно, пространство-время получило бы эвклидову метрику. Одним из следствий этого явилась бы полная относительность временно́й последовательности любой пары событий, ибо исчезло бы различие между пространственно-подобными и временно-подобными интервалами. Тем самым принцип причинности утратил бы всякий смысл. Любую скорость стало бы возможным пребюразовать к бесконечному значению путем над,тежащего выбора инерциальной системы. Такую теорию следует считать, конечно, неприемлемой. Еще одно соображение таково. Примем в формулах (1) и (5) $m_{1}=m_{2}$ и $\gamma_{32}=\gamma_{13}$. Тогда

$$
\begin{gathered}
m_{1}\left(1+\gamma_{12}\right)=m_{3} \gamma_{13}, \\
2 m_{1} \gamma_{13}=m_{3},
\end{gathered}
$$

откуда, исключая $m_{3} / m_{1}$, находим:

$$
\gamma_{12}=2 \gamma_{13}^{2}-1
$$

Следовательно, если $\gamma_{13}<\sqrt{2 / 2}$, т. е. $v_{13}>C$, то $\gamma_{12}<0$. Это значит, что в формуле (36) перед корнем должен стоять двойной знак \pm . Тем самым масса движущегося тела определялась бы только с точностью до знака, без возможности однозначного выбора. Но это не имеет физического смысла.

\section{Заключение}

Нам остается в заключение только дать сравнительную оценку двум вариантам нашего метода. С первого взгляда может показаться, что второй вариант предпочтительнее первого, так как он не нуждается в законах сохранения массы и импульса. На самом деле это преимущество иллюзорно, так как законы сохранения нужны для построения релятивистской динамики. Рано или поздно их все равно следует привлечь к участию в создании теории. Итак, мы должны, если хотим сделать это рано, отдать предпочтение первому варианту. 


\section{ЛИТЕРАТУРА}

1. I g n a tow sky W., Phys. Z., 11, 972 (1910).

2. I g n a tow sky W., Phys. Z., 12, 779 (1911).

3. Frank Ph., Rothe H., Phys. Z., 13, 750 (1912).

4. Frank Ph., R othe H., Ann. Phys., 34, 825 (1911).

5. К а р д П., Изв. АН ЭССР, Физ. Матем., 24, 449 (1975).

6. К а рд П., Изв. АН ЭССР, Физ. Матем., 24, 335 (1975).

7. К а рд П., Изв. АН ӘССР, Физ. Матем., 24, 305 (1975).

Тартуский государственный университет

Поступила в редакцию

$15 / \mathrm{X} \quad 1975$

\section{P. KARD}

\section{ERIRELATIIVSUSTEOORIA POOHJENDAMINE EINSTEINI TEISE POSTULAADITA}

Erirelatiivsusteooria põhjendamiseks piisab (klassikalisest teooriast loobumise kõrval) ainult relatiivsusprintsiibist; valguse kiiruse konstantsust ei ole tarvis eeldada, vaid sellele kui ühele järeldusele jõutakse teooria ülesehitamise käigus. Sel meetodil on kaks võimalikku teostusviisi. Uhes läheb veel vaja massi ja impulsi jäävuse seadusi, teine on sellest eeldusest vaba. Ent inertsiaalteisenduste rühmaomaduste nõue on mõlemal juhul liigne. Selle poolest erineb see meetod teistest varem tuntud meetoditest, milles Einsteini teine postulaat asendatakse rühmapostulaadiga.

\section{P. KARD}

\section{DIE BEGRUNDUNG DER SPEZIELLEN RELATIVITÄTSTHEORIE OHNE ZWEITES POSTULAT EINSTEINS}

Für die Begründung der speziellen Relativitätstheorie genügt neben der Verzichtung auf klassische Theorie nur das Relativitätsprinzip, während das zweite Postulat Einsteins sich als überflüssig erweist. Die Konstanz der Lichtgeschwindigkeit erhält man vielmehr als eine Folge der Theorie im Bau. Es gibt zwei verschiedene Gestaltungen dieser Methode. Einer von diesen liegen obendrein die Erhaltungssätze der Masse und des Impulses zugrunde, die andere ist eben von dieser Voraussetzung frei. Die Forderung der Gruppeneigenschaften der Inertialtransformationen ist aber in beiden Gestaltungen überilüssig. In dieser Hinsicht unterscheidet sich die neue Methode von einigen früher entwickelten Methoden, in welchen das zweite Postulat Einsteins durch die Forderung der Gruppeneigenschaften der Inertialtransformationen ersetzt wird. 